should be further explored in order to have better predictive values of IgAVN outcome and to be incorporated into existing or new classifications, on the basis of which guidelines for the treatment of patients would be developed.

SUPPORT: Croatian Science Foundation project IP-2019-048822.

\section{SINGLE NUCLEOTIDE POLYMORPHISMS OF GENES HMGB1 AND AGER AND ITS ASSOCIATION WITH CLINICAL FEATURES OF IGA VASCULITIS}

\begin{abstract}
${ }^{1}$ Martina Held*, ${ }^{2}$ Mateja Batnozić Varga, ${ }^{1}$ Mario Sestan, ${ }^{2}$ Matej Sapina, ${ }^{1}$ Nastasia Kifer, ${ }^{1}$ Danica Grguric, ${ }^{1}$ Kristina Crkvenac Gornik, ${ }^{1}$ Marijan Frkovic, ${ }^{2}$ Nena Arvaj, ${ }^{2}$ Jasenka Wagner, 'Marija Jelusic. 'University Hospital Centre Zagreb, Department of Pediatrics, University of Zagreb School of Medicine; ${ }^{2}$ University Hospital Centre Osijek, Josip Juraj Strossmayer University of Osijek, Medical Faculty Osijek, Croatia
\end{abstract}

\subsection{6/archdischild-2021-europaediatrics.455}

Introduction IgA vasculitis (IgAV) is a disease in which genetic background also plays an important role. Some small studies have indicated the importance of variants in various non-HLA genes in the manifestation of different disease phenotypes.

Objectives The aim of this research was to investigate single nucleotide polymorphisms (SNPs) of genes HMGB1 and AGER encoding for high mobility group box-1 (HMGB1) and receptor for advanced glycation endproducts (RAGE), both acting as mediators of inflammation, in the susceptibility and clinical features of patients with IgAV.

Methods HMGB1 and RAGE gene polymorphisms were genotyped using a real-time polymerase chain reaction. The presence and frequency of polymorphisms in HMGB1 (rs2249825, rs1045411, rs1060348, rs1412125 and rs41369348) and RAGE (rs1800625, rs1800624, rs2070600 and rs3134940) were determined. Clinical data were collected from database of IgAV patients from two Croatian University Centers for pediatric rheumatology.

Results 81 pediatric IgAV patients were included, of whom 45 were boys and 36 girls, as well as 150 age- and sexmatched healthy controls without any history of autoimmune disease. The median (range) age of IgAV patients was 6.25 (4.60-8.20) years, and among them $71.6 \%$ had joint involvement, $29.62 \%$ had gastrointestinal manifestations, while $27.16 \%$ developed nephritis. The purpuric rash which extended from lower extremities to the trunk, upper extremities and face (generalized rash) was present in $43.20 \%$ of patients and $27.16 \%$ had at least one relapse. Among the analyzed polymorphisms, only in the rs1412125 there was a deviation from the Hardy Weinberger equilibrium. There was no statistically significant association of the analyzed polymorphisms with the IgAV susceptibility, compared to healthy controls. Polymorphism rs2070600 was significantly related with the development of nephritis in IgAV, while rs1412125 was associated with gastrointestinal involvement. The IgAV patients carrying the $\mathrm{T}$ allele (rs2070600) of the AGER had significantly increased risk of nephritis development compared with the IgAV patients with homozygous CC genotype in dominant (OR 4.05, CI 1.09-15.03, p = 0.037) and additive genetic models (OR 3.95, CI 1.16-13.47, $\mathrm{p}=0.049$ ). The minor C allele (rs1412125) of the HMGB1 was found to significantly increase the risk of gastrointestinal involvement in overdominant model with an allelic odd ratio of 2.78 (CI 1.04-7.43, $\mathrm{p}=0.04$ ).
Conclusion Although neither of analyzed HMGB1 and RAGE polymorphisms was not associated with IgAV susceptibility, our results indicated that these polymorphisms may be involved in the pathogenesis of IgAV with possible effect on different disease phenotypes.

SUPPORT: Croatian Science Foundation project IP-2019-048822 .

\section{M2 MACROPHAGE INFILTRATION AND HENOCH- SCHÖNLEIN'S PURPURA NEPHRITIS}

Nastasia Kifer*, Mario Sestan, Martina Held, Domagoj Kifer, Marijan Frkovic, Emina Babarovic, Stela Bulimbasic, Marijana Coric, Alenka Gagro, Gordana Laskarin, Marija Jelusic. University Hospital Centre Zagreb, Department of Paediatrics, University of Zagreb School of Medicine

\subsection{6/archdischild-2021-europaediatrics.456}

Goal Having in mind that macrophages frequently infiltrate injured glomerular and tubulointerstitial tissue, it is possible that the degree and subtype of macrophage infiltration varies depending on the type and severity of renal injury. The aim of this research was to determine macrophage subclasses in renal biopsy specimens of HSPN patients and to analyze their quantity in regard with patients ${ }^{6}$ clinical parameters and histologic features.

Method We performed an immunohistochemical study on renal tissue samples of patients with HSPN, diagnosed by EULAR/PRINTO/PRES criteria and followed for at least 6 months. Patient clinical and laboratory data was retrieved from hospitals' medical records. Renal biopsy samples were marked with antibodies for CD68, iNOS and arginase. The number of immunoreactive cells was counted in each glomerulus by two independent experts.

Results Laboratory and histologic data for 25 patients with HSPN was evaluated in regard with macrophage infiltration of the renal tissue. The median glomerular $\mathrm{M} 1$ and $\mathrm{M} 2$ counts (q1, q3) were $2.3(0.9,12.2)$ and $7.6(4.4,13.9)$, respectively. M1 macrophages were found statistically significantly less frequent in the glomeruli in comparison with M2 macrophages $(p<0.001, b=-0,289 \pm 0.053)$. Collected laboratory data included inflammatory markers and markers of kidney function. There was no significant correlation between M1 and M2 macrophage count and laboratory parameters. Four pathohistological classifications were used: ISKDC, Haas classification, Oxford classification, and SQC classification. Classification stages/total classification scores and all histological variables were evaluated for possible correlation with macrophage count. Statistically significant negative correlations were found between segmental glomerulosclerosis (Oxford classification) and M2 macrophages $(\mathrm{p}=0,001, \mathrm{~b}=-1,050 \pm 0,275)$. An indication of negative correlations was noted between M2 macrophages and segmental sclerosis, and M2 macrophages and adhesions (SQC classification).

Conclusion Glomeruli in HSPN showed predominant M2 polarization of macrophages. M2 macrophage infiltration of glomeruli was highlighted as a possible negative predictor for segmental glomerulosclerosis.

This project is funded by Croatian Science Foundation project PURPURAPREDICTORS IP-2019-04-8822 and University of Rijeka, Croatia grant No. Uni-ri-biomed-18-110. 